\title{
Ulcerative colitis complicated by disseminated intravascular coagulation
}

\author{
S. Muller, ${ }^{1}$ I.M. Chesner, ${ }^{2}$ J. Sheridan ${ }^{3}$ and J. Newman ${ }^{1}$ \\ ${ }^{1}$ Department of Pathology, ${ }^{2}$ Metabolic Unit, ${ }^{3}$ Department of Surgery, East Birmingham Hospital, Bordesley \\ Green East, Birmingham B9 5ST, UK.
}

\begin{abstract}
Summary: We report a case of chronic ulcerative colitis complicated by clinical evidence of disseminated intravascular coagulation and pathological evidence of intestinal ischaemia secondary to venular and capillary fibrin thrombi. This may well represent an example of univisceral Shwartzman reaction occurring in a sensitized target organ.
\end{abstract}

\section{Introduction}

Disseminated intravascular coagulation (DIC) occurs in association with a variety of conditions. It is characterized by the formation of micro-thrombi in small vessels producing ischaemic damage to many organs including lung, heart, kidney, liver and, less commonly, to the gastro-intestinal tract. ${ }^{1}$ Vascular damage to the intestine has been described in a number of circumstances ${ }^{2}$ and may be part of a generalized coagulopathy. ${ }^{3}$ However, the relationship of ulcerative colitis to DIC is not well documented and we describe a case which terminally resulted in fulminant ischaemic colitis with perforation.

\section{Case report}

A 59 year old man presented with a 4-month history of bloody diarrhoea and weight loss. One month before admission, rectal biopsy showed an active colitis with gland distortion, goblet cell depletion, focal ulceration and crypt abscesses (Figure 1). A barium enema performed 4 days before admission showed proctosigmoid colitis.

On examination he was apyrexial, dehydrated and clinically anaemic. Initial investigation revealed a haemoglobin of $6.6 \mathrm{~g} / \mathrm{dl}$, white cell count $13.2 \times 10^{9} / \mathrm{l}$, platelets $150 \times 10^{9} / 1$, sodium $129 \mathrm{mmol} / 1$, potassium $2.7 \mathrm{mmol} / 1$, urea $5.5 \mathrm{mmol} / 1$, creatinine $114 \mu \mathrm{mol} / \mathrm{l}$, albumin $21 \mathrm{~g} / 1$. A plain abdominal film showed mucosal oedema of the left colon and caecal dilatation. He was re-hydrated, transfused and given gentamycin and metronidazole. Blood and stool cultures

Correspondence: J. Newman, F.R.C. Path.

Accepted: 3 February 1987

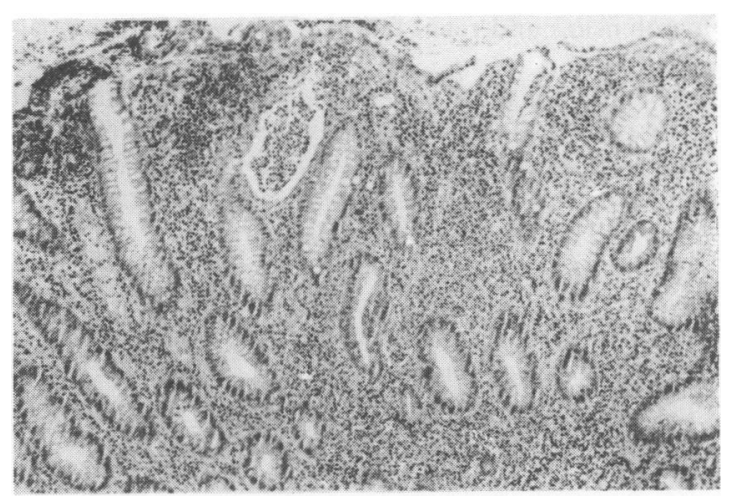

Figure 1 Rectal biopsy showing active chronic colitis with goblet cell depletion, crypt abscess formation, a heavy inflammatory infiltrate and occasional bifid glands $(\mathrm{H} \& \mathrm{E} \times 64)$

were negative. Over a period of 48 hours his rectal bleeding increased and a clotting screen showed a prolonged prothrombin time of 18 seconds (control 13) and partial thromboplastin time (PTT) 49 seconds (control 37); the platelet count fell to $58 \times 10^{9} / 1$. Fibrin degradation products (FDPs) were greater than 20 but less than $40 \mu \mathrm{mol} / 1$ and a clinical diagnosis of DIC was made. He was treated with fresh frozen plasma, platelet transfusion and parenteral hydrocortisone. Radiologically colonic appearances improved. However, his girth increased and rectal bleeding persisted in spite of correction of the thrombocytopaenia. Two days later he developed signs of peritonitis. At laparotomy there was a perforation in the transverse colon with evidence of sub-total colitis. 
Panproctocolectomy was performed and an ileostomy was fashioned. The colon showed large areas of ulceration and necrosis predominantly along the antimesenteric border, together with a $1.5 \mathrm{~cm}$ perforation in the transverse colon. The terminal ileum was normal.

The most striking histological feature was the large numbers of recent fibrin thrombi in many capillaries and veins within all layers of the colonic wall, mesentery, and sinusoids of mesenteric lymph nodes. The large mesenteric arteries and terminal ileal vessels were strikingly normal. All sections were stained to identify and confirm fibrin thrombi. The ulcers extended into the submucosa, with adjacent less severe inflammatory changes superimposed on chronic architectural and glandular damage (Figure 2). In the transverse colon there was transmural ischaemic necrosis with perforation. There was no fibrosis or any other feature to suggest chronic ischaemic damage.

Post-operatively his clotting remained deranged and he developed Gram-negative septicaemia, hypotension and renal failure. He died 18 days postoperatively.

At autopsy there was evidence of left ventricular failure and ischaemic heart disease with an old healed anterior myocardial infarct. Large mesenteric vessels were patent but there was recent portal vein thrombosis and a small liver infarct.

Histology confirmed multi-organ failure with 'shock lung', hepatic centrilobular necrosis, renal tubular necrosis and cerebral micro-haemorrhage. The small bowel showed mucosal autolysis, but no fibrin thrombi were to be seen here or in any other organ.

\section{Discussion}

This case illustrates several interesting features with respect to the role of ischaemia in patients presenting with fulminant colitis, and its relationship to ulcerative colitis.

Submucosal fibrin thrombi are rarely found in inflammatory bowel disease and do not appear to be related to the severity of the colitis. ${ }^{4}$ Vascular thrombosis and necrosis are not features of fulminant ulcerative colitis, but may be seen in the small or large bowel following periods of hypotension. ${ }^{2}$ The term 'ischaemic enterocolitis' has been used to descrihe this phenomenon. Previous studies have shown that it is usually of less than 20 days duration. ${ }^{3,5}$ In our case the insidious onset and relatively long history ( 3 months) together with the rectal biopsy appearances of chronic colitis and the sharp cut-off point at the ileo-caecal valve favour an underlying inflammatory bowel disease rather than an ischaemic enterocolitis.

Macroscopically, ischaemic enterocolitis may

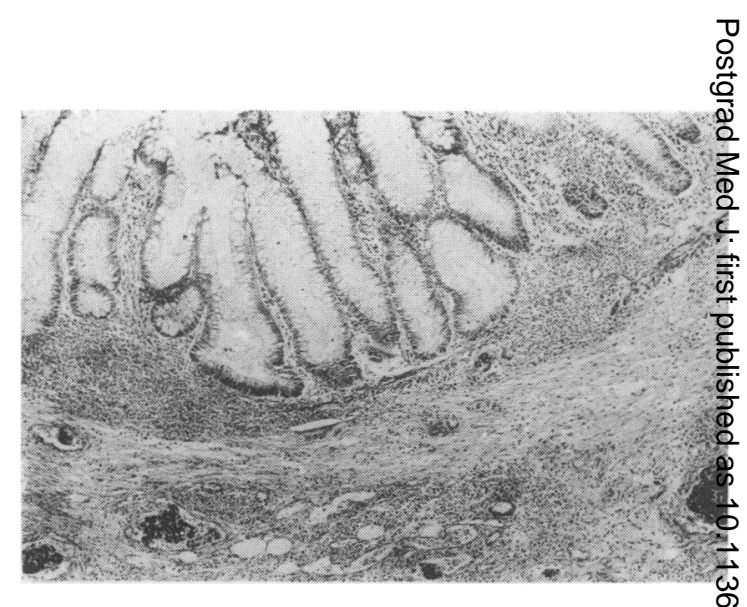

Figure 2 Colon showing distorted and branching glands, residual mucosal chronic inflammation and multiple submucosal fibrin thombi (Picro-Mallory $\times 40$ )

resemble fulminant ulcerative colitis although, in theo former, the lesions are predominantly left-sided ando rectal involvement is uncommon. The histologicaf features are generally different, although on recoveryfrom ischaemic colitis the changes may resemble those of a quiescent inflammatory bowel disease. ${ }^{6}$ In thiso case, there was extensive venous thrombosis withe? subsequent ischaemic necrosis and perforation superimposed on an established ulcerative colitis, 1000 no features of chronic ischaemic damage such muscle atrophy and fibrosis. Furthermore, the recento nature of the fibrin thrombi make low-grade chronic DIC unlikely.

The association of ulcerative colitis and DIC haso๊ only been reported infrequently. ${ }^{7,8} \mathrm{Lo}^{7}$ describes aO young female with a 3-day history of diarrhoea an 6 Gram-negative septicaemia complicated by DIC. On马 review, the history and histology are more suggestive? of an acute infective colitis and the DIC could welp have been secondary to Gram-negative sepsis. The two Japanese cases have no haematological evidence of DIC, and no histological evidence of fibrin thrombis involving the colon.

The haematological evidence of DIC in our case isô presumptive with a normal platelet count on admis sion falling rapidly to $58 \times 10^{9} / 1$. This was associatede with prolongation of prothrombin time and $\mathrm{PI} \mathrm{I}$, and $\mathrm{D}$ a slight elevation of FDPs. Although in active DIC을. FDPs are usually greater than $40 \mu \mathrm{mol} / 1$, levels can fluctuate widely.' Severe acute DIC rarely causes ${ }^{\text {I }}$ significant organ damage unless pre-existing local 0 disease is also present.$^{10}$ In this case, the large intestine was already the site of a chronic inflammatory condi-tion and once DIC supervened, ischaemia compoun ded damage to the colon.

DIC is known to produce ischaemic intestinal necrosis in dogs ${ }^{11}$ and in man. The latter may be one of 
the multi-system manifestations of DIC..$^{3,12}$ However, fibrin thrombi have been seen in both ischaemic and non-ischaemic bowel disease at autopsy and may be non-specific rather than pathognomonic of DIC. DIC appears analogous to the Shwartzman reaction seen in animals following endotoxaemia. ${ }^{13}$ As well as the generalized form, a univisceral or single organ Shwartzman reaction has been described ${ }^{14}$ which may be implicated in the pathogenesis of ischaemic enterocolitis. One mechanism postulated for the univisceral Shwartzman reaction is that of a 'generalized provocation' acting on an already damaged target organ. In our case, the initial thrombotic stimulus provided by DIC acts focally on the inflamed colon producing the univisceral damage seen in the colectomy specimen.

\section{References}

1. Robboy, S.J., Major, M.C., Colman, R.W. \& Minna, J.D. Pathology of disseminated intravascular coagulation (DIC). Analysis of 26 cases. Hum Path 1972, 3: 327 343.

2. McGovern, V.J. \& Goulston, S.J.M. Ischaemic enterocolitis. Gut 1965, 6: 213-220.

3. Whitehead, $R$. Ischaemic enterocolitis: an expression of the intravascular coagulation syndrome. Gut 1971, 12: 912-917.

4. Brandt, L.J., Gomery, P., Mitsudo, S.M., Chandler, M.T. \& Boley, S.J. Disseminated intravascular coagulation in nonocclusive mesenteric ischaemia: the lack of specificity of fibrin thrombi in intestinal infarction. Gastroenterology 1976, 71: 954-957.

5. Shimamura, K., Oka, K., Nakazawa, M. \& Kojima, M. Distribution patterns of microthrombi in disseminated intravascular coagulation. Arch Pathol Lab Med 1983, 107: 543-547.

6. Whitehead, R. The pathology of intestinal ischaemia. Clin Gastroenternl 1972. 1: $613 \quad 6.37$

7. Lo, D. Klebsiella septicaemia, disseminated intravascular coagulation and ulcerative colitis in an Australian Aboriginal. Med J Aust 1971, (i): 1279-1280.

8. Endo, K., Miura, N., Sato, T. et al. Two cases of ulcerative colitis complicating disseminated intravascular coagulation. Nippon Shokakibyo Gakhai Zasshi 1982, 79: $1778-1782$.
Persistence of fibrin thrombi may result from the secondary antithrombin III deficiency which has been demonstrated in patients with ulcerative colitis. ${ }^{15}$ The mechanisms producing reduced antithrombin III levels include increased catabolism ${ }^{16}$ and increased loss in a protein-losing enteropathy. The combined effects of these two mechanisms may result in significantly reduced antithrombin III activity and subsequent failure to neutralize thrombin formation.

\section{Acknowledgements}

The authors thank Mr R.T.J. Holl-Allen for allowing us to report this case, Dr A.B. Price for helpful comments on the histology, and Mr M.J. Chard, Mr C. Davies and Miss E. White lor technical and secretarial assistance.

9. Mason, D.Y., Heenan, P.J. \& Whitehead, R. Disseminated intravascular thrombosis as the cause of ischaemic enterocolitis. Med J Aust 1976, (i): 440-442.

10. Mant, M.J. \& King, E.G. Severe acute disseminated intravascular coagulation. A reappraisal of its pathophysiology, clinical significance and therapy based on 47 patients. Am J Med 1979, 67: 557-563.

11. Kondo, M., Yoshikawa, T., Takemura, S., Yokoe, N., Kawai, K. \& Masuda, M. Haemorrhagic necrosis of the intestinal mucosa associated with disseminated intravascular coagulation. Digestion 1978, 17: 38-45.

12. Margaretten, W. \& McKay, D.G. Thrombotic ulcerations of the gastrointestinal tract. Arch Intern Med 1971, 123: $250-253$.

13. Shwartzman, G. A new phenomenon of local skin reactivity to B typhosus culture filtrate. Proc Soc Exp Biol Med 1928, 25: 560-561.

14. Mori, W. The Shwartzman reaction: a review including clinical manifestations and proposal for a univisceral or single organ third type. Histopathology 1981, 5: 113-126.

15. Lake, A.M., Stauffer, J.Q. \& Stuart, M.J. Haemostatic alterations in inflammatory bowel disease. Am J Dig Dis 1978, 23: 897-902.

16. Knot, E.A.R., ten Cate, J.W., Bruin, T., Iburg, A.H.C. \& Tytgat, G.N.J. Antithrombin III metabolism in two colitis patients with acquired antithrombin III deficiency. Gastroenterology 1985, 89: 421-425. 